\title{
6 \\ LEVERAGING INFORMATION TECHNOLOGY FOR ORGANIZATIONAL RESILIENCE IN DESIGN OF COMPLEX PRODUCTS: A Case Study
}

\author{
Rajeev Lal \\ Infotech Enterprises Ltd. \\ Hyderabad, India \\ Avinash Samrit \\ Infotech Enterprises Ltd. \\ Singapore
}

\begin{abstract}
Infotech Enterprises Ltd. provides IT and engineering design services, and is certified for CMMI level 5, ISO 9000-2000, BS 7799, and AS9100. One of our major customers is a leading aero engines design and manufacturing company in the United States. The customer designs and manufactures jet engines, the most sophisticated breakthrough of the $20^{\text {th }}$ century. Around 2001, this customer initiated work on development of an engineering design framework for constant innovation, greater productivity, and better success and profitability. It also aimed at resilience against serious loss of knowledge and experience as employees retire or leave suddenly. It is important for organizations to have the resilience to muster up the required size and skill mix of teams based on need, sometimes at a very short notice. An important component for this resilience is a framework that enables new entrants in the projects, whether employees or companies to which work is assigned, to quickly come up to speed as well as be able to do their work correctly. The framework consists of knowledge repository, design process, visual representation, quick navigation, standardization, and easy workflow.

One of our large customers, a leading aero engine design and manufacturing company, under relentless pressure from customers and competitors, had been trying a radical change in the engineering design process. We started working on a solution jointly in 2002. The challenge was to develop a framework that is easy to learn and use, and has the capability for global deployment. We set up a team of IT specialists who were adept at grasping new technologies quickly. The first decision taken was to use a web browser
\end{abstract}

Please use the following format when citing this chapter:

Lal, Rajeev, Samrit, Avinash, 2006, in International Federation for Information Processing (IFIP),

Volume 206, The Transfer and Diffusion of Information Technology for Organizational Resilience, eds. B. Donnellan, Larsen T., Levine L., DeGross J. (Boston: Springer), pp. 93-99. 
interface that is commonly used. A standard document storage mechanism based on XML was decided upon. A unique and very convenient method of quickly navigating to the desired point from within a large diagram was developed on the browser. The first version was ready for deployment in 18 months.

A 30 percent improvement in engineering design productivity had taken place once the implementation stabilized. By that time, all 4,000 design engineers in the organization were using the framework. Extracts relating to work outsourced to external companies were passed on to the suppliers, resulting in improvements. This increased organizational resilience in effectively managing varying levels of manpower resource requirement. Other companies of the group are now initiating similar frameworks. IT tools, especially web technologies and connectivity, now make it passible for an organization to implement such a framework within and across engineering design teams, both internal and external. IT should be leveraged by large companies to attain and maintain competitive leadership through resilience.

Keywords Organizational resilience, knowledge repository, engineering design productivity, competitive leadership

\section{INTRODUCTION}

Infotech Enterprises Ltd. provides information technology and engineering design services, and is certified for CMMI level 5, ISO 9000-2000, BS 7799, and AS9100. One of our major customers is a leading aero engines design and manufacturing company in the United States. The customer designs and manufactures jet engines, the most sophisticated breakthrough of the $20^{\text {th }}$ century. The product design process is highly manpower intensive, with a combination of creativity and diligent analysis and validation. Around 2001, this customer initiated work on development of an engineering design framework for constant innovation, greater productivity, and better success and profitability. It also aimed at resilience against serious loss of knowledge and experience as employees retire or leave suddenly. All parts of the design activity that did not involve pushing the envelope were standardized. To build on lessons from past experiences, the organization wanted a system to identify and convert individual expertise, skills, and experience into organizational resources.

The customer found good results in meeting the need to shorten development time, use fewer resources and have fewer quality problems using a framework that it called engineering standard work (ESW). The framework enabled robust results under conditions of stress and change. The stress is caused by difficulties in quickly generating new strategic options (e.g., effectively deploying large teams at short notice and imparting them with knowledge traditionally documented and stored on the desks of individual practitioners). A major facilitator of this resilience is the ability to outsource part of the work. This ability enables use of external help in a profitable manner. Lack of it results in the organization sometimes spending more effort in getting work done from outside than it would have spent in doing it internally.

ESW expedited controlled innovation, and brought about a radical change in the engineering design process. For producing good results, engineers require subject 
knowledge in product domain and engineering disciplines like modeling, analysis of various types (structural, dynamic, thermal), and product testing. ESW integrated the disciplinary functions and formalized the design, development, and review processes.

The benefits of ESW were clearly noticed wherever it was introduced. The customer, however, had no mechanism in place to ensure that it was used, sustained, and improved. Methodologies were locally documented in different formats. Lessons learned for not captured formally. If the department chief or a few experts left, there was trouble at hand. It was decided to use technology as a tool for capturing and organizing organizational knowledge and diffusing it.

\section{FRAMEWORK DEFINED}

The following elements were identified in ESW by the customer:

- Workflow maps

- Tools and methods

- Design criteria

- Design standards

- Lessons learned

- Practitioner proficiency assessments

Workflow maps depicted the design process at the system, module, and part levels. The maps captured inputs, outputs, and dependencies between tasks and activities.

An activity page prescribed each discrete task on the map. The page contained a task description, work instructions and tools, the range of applicability of each tool and method, required inputs and outputs (from whom and to whom), design criteria, documentation and sign-offs, design standards, and a resource plan.

Tools and methods included "how to" work instructions, their range of applicability, modeling standards, and validation.

Lessons learned emphasized constant improvement of ESW as better tools, methodologies, and work instructions evolved.

An assessment found that for every $\$ 1$ spent on ESW, the customer achieved a cost savings of nearly $\$ 4$.

\section{RESILIENT FRAMEWORK}

The customer used Infotech's services to develop an IT solution that would enable the implementation and diffusion of ESW across its organization. We examined the possibility of a ready-made solution, but could not identify one that would meet the customers' requirements. Functionality required in the solution consisted of the following:

- Knowledge repository: A database to serve as a repository for standards, guidelines, etc. There should be a process to add, change, or remove items in the repository with appropriate authorization and access control. 
- Design process: A convenient way of specifying and design process. Each design process will be a subset taken out of the options available in the organization.

- Visual representation: A flow chart or a diagram is the easiest way to communicate a process and its components. Some flow charts were already in use. If the complete engineering design process can be put in a flow diagram, it can be easily understood by any one required to use it.

- Quick navigation: In a large flow diagram, there should be a quick way to reach a selected section. Within the chosen section, it should be easily possible to access all of the related design processes and documents.

- Standardization: As the system will be used by a large number of people whose work will be integrated in various ways, it is necessary to standardize the process representation, the documents, and the norms for design.

- Easy workflow: Review and approval is an important component of a robust design process. It should be possible to store results of the work done, and for a specialist or manager to review it conveniently.

\section{VIRTUAL SOLUTION LEVERAGING IT}

We started working on a solution jointly with the customer in 2002. The design team was already using computers and design software. The challenge was to develop a framework that is easy to learn and use, and that has the capability for global deployment. We set up a team of IT specialists who were adept at grasping new technologies quickly and in finding innovative ways to use them in solutions. The first decision taken was to use a browser interface, which is commonly used and hence does not require any training to start. While the concept of the framework was under discussion for some time, there was no detailed description or specification on which the IT solution could be developed.

The entire user interface was developed in industry standard HTML and XML with standard client-side scripting. Despite the flexibility and ease of development in image based interactive user interface, Java applets were not used in order to comply with the security standards.

The Java application implemented model view controller (MVC) architecture, providing segregation of business logic and presentation layer. This has higher maintainability and extensibility.

Microsoft Visio was the incumbent tool used by the engineers for drawing the process flow maps. It was decided to continue with the same tool for flow maps. An innovative add-on, Remote Visio, was developed to access and edit the Visio map from the central servers with the security mechanism built into it. Visio templates with builtin macros to capture the process flow map coordinates and underlying data were developed to provide the required task-specific data to the Java application. 
Documentum is used in this implementation for document management. Web based ESW leverages the powerful features of Documentum viz document lifecycle and document workflow by integrating them with the web-based application using Java interfaces. Users having creator, reviewer, or approver status can create, review, and approve the technical content respectively.

Although the documents are maintained in XML format and rendered on a web browser, the look and feel of the documents were maintained identical to that of the customer's recent Microsoft Word template. This not only improved the user acceptance but also reduced the user training effort.

Various engineers created the standard work technical content (activity pages, work instructions, etc.) over more than 30 years. With the advent of new processes, materials, and practices, these were updated many times. The content existed in various file formats following different templates. A set of data migration tools using Visual Basic, Pro $\mathrm{C}$, and $\mathrm{VC}++$ were developed to import this technical content into the standard XML templates.

The first version was available in 18 months from the date of starting work and was implemented as per schedule. The iterative method of finalizing specifications resulted in continuous flow of new requirements and changes. It was therefore decided to freeze specifications at some stage so that the original schedule for deployment could be maintained. Requirements and specifications were collated for subsequent changes after a roll out of the first fully tested version.

\section{APPLICATION PERFORMANCE}

Web based applications often have challenges in the area of application performance. Some of the challenges we faced were

- Delayed loading of the flow maps on the browser: The workflow maps are viewed in two sections, one section provides a bird's eye miniature view with a tracker for locating the user's view and the other section a zoomed in flow map that can be scrolled to view and get the required information. The initial version of the application created the miniature view, a resource intensive task, on the fly. The miniature map is now created at the time of saving the Visio map by the creator. This is stored along with the "real" map.

- Overall slow response: On analysis it was found that whichever page referred to LDAP, which has more than 150,000 users, was slow. Some tweaking of the code and caching algorithms helped in solving this problem.

Documentum settings and workflow options were found to have a significant bearing on the performance. In a trade-off between some "nice-to-have" features and performance, some low priority not-so-important features were restricted.

\section{IMPLEMENTATION AND SUCCESS}

The deployment was driven by high customer initiative and was effectively done. On a monthly basis, the organization began to report their progress to senior manage- 
ment. Finally, a central ESW office was created to conduct ESW process assessments, provide IT infrastructure support and a framework for the ESW documentation process, and establish a robust ESW content creation and revision process.

An audit was conducted by the customer after the implementation had stabilized concluding that a 30 percent improvement in engineering design productivity had taken place. By that time, all 4,000 engineers in the organization were using the framework. Improvements had occurred because

- Engineers were able to easily locate the standard method to be followed for a new design or design improvement

- The reduction in rework as all instructions were available clearly in advance before the work was started

- Quick information was provided to managers and subject matter experts to check the results of work done and provide timely feedback by e-mail and a workflow process

- $\quad$ Ease in storing the results of the work done for checking and confirmation

No instances of engineers in a group referring to different versions of technical content were reported. A major cause of concern was thus addressed. Engineers with relatively less experience were able to take up tasks that were otherwise performed by highly experienced engineers only.

The major benefit derived by the organization was that knowledge, which was otherwise maintained by and confined to experienced personnel, became easily available to all engineers almost instantaneously. Knowledge was retained in the organization even if the person who created it went away. The framework provides a dynamic platform for innovation through continuous improvement. The quality of the first result is much higher because very few assumptions were made; detailed information is available for the engineer at the time of starting work. Extracts relating to work outsourced to external companies were passed on to the suppliers, resulting in improvements. The organization's capability to outsource work is greatly enhanced, and managing this outsourcing became easier and less prone to risk.

\section{SECURITY}

Virtual business and virtual work require vigilant attention. Hundreds of users are developing discussing and completing their work through e-mail, collaboration tools, etc. Unauthorized people may peer into crucial data.

The customer already had a robust security and access control mechanism for its intranet, and it was incorporated in the ESW application. User authentication and access roles have been implemented in light weight directory access protocol (LDAP). The application has been integrated with Netscape LDAP server. The super user manages the access control list (ACL) using a web-based interface. The LDAP access control list works seamlessly with Documentum as well. Hence security and access control are centralized and robust. 


\section{EVOLVING SOLUTION}

The use of ESW generated suggestions for enhancements and improvements. Some of the improvements in later version include

- Provision for integrating lessons learned and suggestions into the mainstream workflow

- Reports for process performance measurement

- Resource planning feature

- ESW usage measurement

- Further refinement of Remote Visio

\section{CONCLUSION}

IT tools, especially web technologies and connectivity, now make it possible for an organization to implement a framework like ESW within and across engineering design teams. The ESW framework for the product design and engineering process leads to organizational resilience in effectively managing varying levels of manpower resource requirements and meeting the challenge of sudden loss of expertise. It enables optimum cost and schedule in product development.

Based on the success achieved by the customer, other companies in its group have initiated similar exercises. Our other large customers have also shown keen interest in developing and implementing similar frameworks. Such implementations can be leveraged by large companies to attain and maintain competitive leadership through organizational resilience.

\section{About the Authors}

Rajeev Lal is President, Strategic \& New Business Initiatives, at Infotech Enterprises Ltd., Hyderabad, India. Founded in 1991, Infotech (www.infotechsw.com) is a leading provider of engineering, software, and geospatial services to manufacturing, utility, and government verticals worldwide. Infotech currently employs about 4,000 people across 17 global locations. Rajeev has been in information services for over 33 years and has worked at a number of renowned companies. In Infotech he led the initiative for successful certification of the software development business to ISO 9001 in 1999, SEI CMM Level 5 in 2002, and CMMi Level 5 in 2003. He is chairman of the company's Security Forum. He is a Fellow of The Institution of Engineers (India) and has been a member of American Management Association. Rajeev can be reached at rajeevl@infotechsw.com.

Avinash Samrit has a bachelor's degree in mechanical engineering, a top ranker in his class. Avinash has been associated with the IT industry for more than 13 years. He has played various technical and leadership roles in IT organizations, with eBusiness portals and relational databases being his areas of technical expertise. Avinash joined Infotech Enterprises Ltd. in 2000, managing large teams and complex IT projects for some Fortune 100 clients. Avinash is currently Assistant General Manager responsible for Singapore operations of Infotech. He has been the project manager for the Engineering Standard Work IT solution developed by Infotech. He can be reached at avinashs@infotechsw.com. 